\title{
On relative separability in hypergraphs of models of theories
}

\author{
B.Sh. Kulpeshov, S.V. Sudoplatov
}

\begin{abstract}
In the paper, notions of relative separability for hypergraphs of models of a theory are defined. Properties of these notions and applications to ordered theories are studied: characterizations of relative separability both in a general case and for almost $\omega$-categorical quite o-minimal theories are established.
\end{abstract}

Keywords: hypergraph of models, elementary theory, separability, relative separability.

Hypergraphs of models of a theory are related to derivative objects allowing to obtain an essential structural information both on theories themselves and related semantical objects including graph ones [1, 2, 3, 4, 5, 6, 7, 8].

In the present paper, notions of relative separability for hypergraphs of models of a theory are defined. Properties of these notions and applications to ordered theories are studied: characterizations of relative separability both in a general case and for almost $\omega$-categorical quite o-minimal theories are established.

\section{Preliminaries}

Recall that a hypergraph is a pair of sets $(X, Y)$, where $Y$ is some subset of the Boolean $\mathcal{P}(X)$ of the set $X$.

Let $\mathcal{M}$ be some model of a complete theory $T$. Following [5], we denote by $H(\mathcal{M})$ a family of all subsets $N$ of the universe $M$ of $\mathcal{M}$ that are universes of elementary submodels $\mathcal{N}$ of the model $\mathcal{M}: H(\mathcal{M})=\{N \mid \mathcal{N} \preccurlyeq \mathcal{M}\}$. The pair $(M, H(\mathcal{M}))$ is called the hypergraph of elementary submodels of the model $\mathcal{M}$ and denoted by $\mathcal{H}(\mathcal{M})$.

For a cardinality $\lambda$ by $H_{\lambda}(\mathcal{M})$ and $\mathcal{H}_{\lambda}(\mathcal{M})$ are denoted restrictions for $H(\mathcal{M})$ and $\mathcal{H}(\mathcal{M})$ respectively on the class of elementary submodels $\mathcal{N}$ of models $\mathcal{M}$ such that $|N|<\lambda$.

By $\mathcal{H}_{p}(\mathcal{M})$ we denote the restriction of the hypergraph $\mathcal{H}_{\omega_{1}}(\mathcal{M})$ on the class of elementary submodels $\mathcal{N}$ of the model $\mathcal{M}$ that are prime over finite sets. Similarly by $H_{p}(\mathcal{M})$, is denoted the corresponding restriction for $H_{\omega_{1}}(\mathcal{M})$.

Definition 1.1 [5, 9]. Let $(X, Y)$ be a hypergraph, $x_{1}, x_{2}$ be distinct elements of $X$. We say that the element $x_{1}$ is separated or separable from the element $x_{2}$, or $T_{0}$-separable if there is $y \in Y$ such that $x_{1} \in y$ and $x_{2} \notin y$. The elements $x_{1}$ and $x_{2}$ are called separable, $T_{2}$-separable, or Hausdorff separable if there are disjoint $y_{1}, y_{2} \in Y$ such that $x_{1} \in y_{1}$ and $x_{2} \in y_{2}$.

Theorem 1.2 [5]. Let $\mathcal{M}$ be an $\omega$-saturated model of a countable complete theory $T$, a and $b$ be elements of $\mathcal{M}$. The following are equivalent:

(1) the element a is separable from the element $b$ in $\mathcal{H}(\mathcal{M})$; 
(2) the element $a$ is separable from the element $b$ in $\mathcal{H}_{\omega_{1}}(\mathcal{M})$;

(3) $b \notin \operatorname{acl}(a)$.

Theorem 1.3 [5]. Let $\mathcal{M}$ be an $\omega$-saturated model of a countable complete theory $T$, a and $b$ be elements of $\mathcal{M}$. The following are equivalent:

(1) the elements $a$ and $b$ are separable in $\mathcal{H}(\mathcal{M})$;

(2) the elements $a$ and $b$ are separable in $\mathcal{H}_{\omega_{1}}(\mathcal{M})$;

(3) $\operatorname{acl}(a) \cap \operatorname{acl}(b)=\varnothing$.

Corollary 1.4 [5]. Let $\mathcal{M}$ be an $\omega$-saturated model of a countable complete theory $T$, $a$ and $b$ be elements of $\mathcal{M}$, and there exists the prime model over a. The following are equivalent:

(1) the element a is separable from the element $b$ in $\mathcal{H}(\mathcal{M})$;

(2) the element $a$ is separable from the element $b$ in $\mathcal{H}_{\omega_{1}}(\mathcal{M})$;

(3) the element a is separable from the element $b$ in $\mathcal{H}_{p}(\mathcal{M})$;

(4) $b \notin \operatorname{acl}(a)$.

Corollary 1.5 [5]. Let $\mathcal{M}$ be an $\omega$-saturated model of a countable complete theory $T$, a and $b$ be elements of $\mathcal{M}$, and there exist the prime models over a and $b$ respectively. The following are equivalent:

(1) the elements $a$ and $b$ are separable in $\mathcal{H}(\mathcal{M})$;

(2) the elements $a$ and $b$ are separable in $\mathcal{H}_{\omega_{1}}(\mathcal{M})$;

(3) the elements $a$ and $b$ are separable in $\mathcal{H}_{p}(\mathcal{M})$;

(4) $\operatorname{acl}(a) \cap \operatorname{acl}(b)=\varnothing$.

Definition 1.6 [5]. Let $(X, Y)$ be a hypergraph, $X_{1}, X_{2}$ be disjoint nonempty subsets of the set $X$. We say that the set $X_{1}$ is separated or separable from the set $X_{2}$, or $T_{0}$-separable if there is $y \in Y$ such that $X_{1} \subseteq y$ and $X_{2} \cap y=\varnothing$. The sets $X_{1}$ and $X_{2}$ are called separable, $T_{2}$-separable, or Hausdorff separable if there are disjunct $y_{1}, y_{2} \in Y$ such that $X_{1} \subseteq y_{1}$ and $X_{2} \subseteq y_{2}$.

By using proofs of theorems 1.2 and 1.3 , the following generalizations of these theorems are established.

Theorem 1.7 [5] Let $\mathcal{M}$ be a $\lambda$-saturated model of a complete theory $T, \lambda \geq \max \{|\Sigma(T)|, \omega\}$, $A$ and $B$ be nonempty sets in $\mathcal{M}$ having the cardinalities $<\lambda$. The following are equivalent:

(1) the set $A$ is separable from the set $B$ in $\mathcal{H}(\mathcal{M})$;

(2) the set $A$ is separable from the set $B$ in $\mathcal{H}_{\lambda}(\mathcal{M})$;

(3) $\operatorname{acl}(A) \cap B=\varnothing$.

Theorem 1.8 [5] Let $\mathcal{M}$ be a $\lambda$-saturated model of a complete theory $T, \lambda \geq \max \{|\Sigma(T)|$, $\omega\}, A B$ be nonempty sets in $\mathcal{M}$ having the cardinalities $<\lambda$. The following are equivalent:

(1) the sets $A$ and $B$ are separable in $\mathcal{H}(\mathcal{M})$;

(2) the sets $A$ and $B$ are separable in $\mathcal{H}_{\lambda}(\mathcal{M})$;

(3) $\operatorname{acl}(A) \cap \operatorname{acl}(B)=\varnothing$.

We obtain by analogy with corollaries 1.4 and 1.5 
Corollary 1.9 [5]. Let $\mathcal{M}$ be an $\omega$-saturated model of a small theory $T, A$ and $B$ be finite nonempty sets in $\mathcal{M}$. The following are equivalent:

(1) the set $A$ is separable from the set $B$ in $\mathcal{H}(\mathcal{M})$;

(2) the set $A$ is separable from the set $B$ in $\mathcal{H}_{\omega_{1}}(\mathcal{M})$;

(3) the set $A$ is separable from the set $B$ in $\mathcal{H}_{p}(\mathcal{M})$;

(4) $\operatorname{acl}(A) \cap B=\varnothing$.

Corollary 1.10 [5]. Let $\mathcal{M}$ be an $\omega$-saturated model of a small theory $T, A$ and $B$ be finite nonempty sets in $\mathcal{M}$. The following are equivalent:

(1) the sets $A$ and $B$ are separable in $\mathcal{H}(\mathcal{M})$;

(2) the sets $A$ and $B$ are separable in $\mathcal{H}_{\omega_{1}}(\mathcal{M})$;

(3) the sets $A$ and $B$ are separable in $\mathcal{H}_{p}(\mathcal{M})$;

(4) $\operatorname{acl}(A) \cap \operatorname{acl}(B)=\varnothing$.

The following proposition extends Theorem 1.8 with an additional criterion.

Proposition 1.11 Let $T$ be a theory, $\mathcal{M} \models T, \emptyset \neq A \subseteq M, \emptyset \neq B \subseteq M, \mathcal{M}$ be $|A \cup B|^{+}$saturated. Then $A$ and $B$ are separable from each other in $\mathcal{H}(\mathcal{M})$ if and only if the following conditions hold:

(1) $\operatorname{acl}(A) \cap \operatorname{acl}(B)=\emptyset$;

(2) For any isolated type $p \in S_{1}(\emptyset), p(\mathcal{M}) \backslash \operatorname{acl}(A) \neq \emptyset$ and $p(\mathcal{M}) \backslash \operatorname{acl}(B) \neq \emptyset$.

Proof of Proposition 1.11. If $A$ and $B$ are separable from each other in $\mathcal{H}(\mathcal{M})$ then by Theorem 1.8 we have $\operatorname{acl}(A) \cap \operatorname{acl}(B)=\emptyset$. If there is an isolated type $p \in S_{1}(\emptyset)$ such that $p(M) \subseteq \operatorname{acl}(A)$ then there is $\mathcal{M}_{2} \prec \mathcal{M}$ with $B \subseteq M_{2}$ and $p(\mathcal{M}) \cap M_{2}=\emptyset$, i.e. $p$ is not realized in $\mathcal{M}_{2}$. Similarly, $p(\mathcal{M}) \nsubseteq \mathbb{a c l}(A)$.

If the conditions (1), (2) hold then $A$ and $B$ are separable from each other in $\mathcal{H}(\mathcal{M})$ by Theorem 1.8,

Recall that a subset $A$ of a linearly ordered structure $M$ is called convex if for any $a, b \in A$ and $c \in M$ whenever $a<c<b$ we have $c \in A$. A weakly o-minimal structure is a linearly ordered structure $M=\langle M,=,<, \ldots\rangle$ such that any definable (with parameters) subset of the structure $M$ is a union of finitely many convex sets in $M$.

In the following definitions $M$ is a weakly o-minimal structure, $A, B \subseteq M, M$ be $|A|^{+}$saturated, $p, q \in S_{1}(A)$ be non-algebraic types.

Definition 1.12 12 We say that $p$ is not weakly orthogonal to $q\left(p \not \not^{w} q\right)$ if there exist an $A$-definable formula $H(x, y), \alpha \in p(M)$ and $\beta_{1}, \beta_{2} \in q(M)$ such that $\beta_{1} \in H(M, \alpha)$ and $\beta_{2} \notin H(M, \alpha)$.

Definition 1.13 [13] We say that $p$ is not quite orthogonal to $q\left(p \not \swarrow^{q} q\right)$ if there exists an $A$-definable bijection $f: p(M) \rightarrow q(M)$. We say that a weakly o-minimal theory is quite o-minimal if the notions of weak and quite orthogonality of 1-types coincide.

In the work [14 the countable spectrum for quite o-minimal theories with non-maximal number of countable models has been described:

Theorem 1.14 Let $T$ be a quite o-minimal theory with non-maximal number of countable models. Then $T$ has exactly $3^{k} \cdot 6^{s}$ countable models, where $k$ and $s$ are natural numbers. Moreover, for any $k, s \in \omega$ there exists a quite o-minimal theory $T$ having exactly $3^{k} \cdot 6^{s}$ countable models. 
Realizations of these theories with a finite number of countable models are natural generalizations of Ehrenfeucht examples obtained by expansions of dense linear orderings by a countable set of constants, and they are called theories of Ehrenfeucht type. Moreover, these realizations are representative examples for hypergraphs of prime models [1, 3, 5].

\section{Relative separability in hypergraphs of models of theories}

Observe that since by Theorem 1.8 and Corollary 1.10 separability of sets $A$ and $B$ in hypergraphs $H(\mathcal{M})$ is possible only when $\operatorname{acl}(A) \cap \operatorname{acl}(B)=\varnothing$, such a separability doesn't hold when $\operatorname{acl}(\emptyset) \neq \emptyset$. Thus, it is natural to consider the following notions of relative separability.

Definition 2.1 Let $(X, Y)$ be a hypergraph, $x_{1}, x_{2}$ be distinct elements of $X, Z \subset X$, $x_{2} \notin Z$. We say that the element $x_{1}$ is $Z$-separated or $Z$-separable from the element $x_{2}$, or $\left(T_{0}, Z\right)$-separable if there is $y \in Y$ such that $x_{1} \in y \cup Z$ and $x_{2} \notin y$. In this case the set $y$ is called $Z$-separating $x_{1}$ from $x_{2}$. At the additional condition $x_{1} \notin Z$ the elements $x_{1}$ and $x_{2}$ are called $Z$-separable, $\left(T_{2}, Z\right)$-separable, or Hausdorff $Z$-separable if there are $y_{1}, y_{2} \in Y$ such that $\left(y_{1} \cap y_{2}\right) \backslash Z=\varnothing, x_{1} \in y_{1}$ and $x_{2} \in y_{2}$.

Let $X_{1}, X_{2}$ be nonempty subsets of the set $X,\left(X_{1} \cap X_{2}\right) \backslash Z=\emptyset, X_{2} \nsubseteq Z Z$. We say that the set $X_{1}$ is $Z$-separated or $Z$-separable from the set $X_{2}$, or $\left(T_{0}, Z\right)$-separable if there is $y \in Y$ such that $X_{1} \subseteq y \cup Z$ and $\left(X_{2} \cap y\right) \backslash Z=\varnothing$. At the additional condition $X_{1} \nsubseteq Z$ the sets $X_{1}$ and $X_{2}$ are called $Z$-separable, $\left(T_{2}, Z\right)$-separable, or Hausdorff $Z$-separable if there are $y_{1}, y_{2} \in Y$ such that $\left(y_{1} \cap y_{2}\right) \backslash Z=\varnothing, X_{1} \subseteq y_{1} \cup Z$ and $X_{2} \subseteq y_{2} \cup Z$.

Remark 2.2 1. The notions of separability given in Section 1 correspond $Z$-separability for $Z=\varnothing, X_{1} \neq \varnothing, X_{2} \neq \varnothing$.

2. If $X_{2} \subseteq Z$ then the set $X_{2}$ also can be assumed $Z$-separable from $X_{1}$, although there is no reason to say on real separability of elements of the set $X_{2}$ from $X_{1}$.

For a tuple $\bar{a}$ and a set $Z$ we denote by $\bar{a} Z$ the union of the set $Z$ with the set of all elements containing in $\bar{a}$.

The following theorem modifies Theorem 1.2, and it is a generalization of the theorem for $\operatorname{acl}(\varnothing)=\varnothing$.

Theorem 2.3 Let $\mathcal{M}$ be an $\omega$-saturated model of a countable complete theory $T, Z$ be the algebraic closure of some finite set in $\mathcal{M}, a$ and $b$ be elements of $\mathcal{M}, b \notin Z$. The following are equivalent:

(1) the element a is $Z$-separable from the element $b$ in $H(\mathcal{M})$ by some set $y$ from $H(\mathcal{M})$ containing $Z$;

(2) the element $a$ is $Z$-separable from the element $b$ in $H_{\omega_{1}}(\mathcal{M})$ by some set $y$ from $H_{\omega_{1}}(\mathcal{M})$ containing $Z$;

(3) $b \notin \operatorname{acl}(a Z)$.

Proof. The implications $(2) \Rightarrow(1)$ and $(1) \Rightarrow(3)$ are obvious (clearly, if $b \in \operatorname{acl}(Z \cup\{a\})$ then $b$ belongs to any model $\mathcal{N} \preccurlyeq \mathcal{M}$ containing $Z \cup\{a\})$.

To prove the implication (3) $\Rightarrow(2)$ we need the following lemma.

Lemma 2.4 Let $\bar{a}$ be a tuple, $B$ be a finite set for which $(\operatorname{acl}(\bar{a} Z) \cap B) \backslash Z=\varnothing$, and $\varphi(x, \bar{a})$ be some consistent formula. Then there is an element $c \in \varphi(\mathcal{M}, \bar{a})$ such that $(\operatorname{acl}(\bar{a} c Z) \cap B) \backslash Z=$ $\varnothing$. 
Proof of Lemma 2.4. If $\varphi(\mathcal{M}, \bar{a}) \cap Z \neq \varnothing$ then there is nothing to prove since as $c$ we can take an arbitrary element of $\varphi(\mathcal{M}, \bar{a}) \cap Z$.

Suppose that $\varphi(\mathcal{M}, \bar{a}) \cap Z=\varnothing$. By compactness and using consistent formulas $\varphi^{\prime}(x, \bar{a})$ with the condition $\varphi^{\prime}(x, \bar{a}) \vdash \varphi(x, \bar{a})$ instead of $\varphi(x, \bar{a})$, it is sufficiently to prove that for any $d \in B \backslash Z$ and the finite set of formulas $\psi_{1}(x, \bar{a}, y), \ldots, \psi_{n}(x, \bar{a}, y)$ with the condition

$$
\psi_{i}(x, \bar{a}, y) \vdash \varphi^{\prime}(x, \bar{a}) \wedge \forall x\left(\varphi^{\prime}(x, \bar{a}) \rightarrow \exists^{=k_{i}} y \psi_{i}(x, \bar{a}, y)\right)
$$

for some natural $k_{i}, i=1, \ldots, n$, there is an element $c \in \varphi^{\prime}(\mathcal{M}, \bar{a})$ such that

$$
\models \bigwedge_{i=1}^{n} \neg \psi_{i}(c, \bar{a}, d) .
$$

Assume the contrary: for any $c \in \varphi^{\prime}(\mathcal{M}, \bar{a})$ there is $i$ such that $\models \psi_{i}(c, \bar{a}, d)$. Then the formula $\chi(x, \bar{a}, y) \rightleftharpoons \bigvee_{i=1}^{n} \psi_{i}(x, \bar{a}, y)$ satisfies the following condition: for any $c \in \varphi^{\prime}(\mathcal{M}, \bar{a})$, $\models \chi(c, \bar{a}, d)$ and $\chi(c, \bar{a}, y)$ has finitely many, no more than $m=k_{1}+\ldots+k_{n}$ solutions. Consequently, the formula

$$
\theta(\bar{a}, y) \rightleftharpoons \exists x\left(\chi ( x , \overline { a } , y ) \wedge \forall z \left(\left(\varphi^{\prime}(z, \bar{a}) \rightarrow(\chi(x, \bar{a}, y) \wedge \chi(z, \bar{a}, y))\right)\right.\right.
$$

satisfies $d$ and has no more than $m$ solutions. This fact contradicts the condition $d \notin \operatorname{acl}(\bar{a} Z)$.

Assuming that $b \notin \operatorname{acl}(a Z)$, we construct by induction a countable model $\mathcal{N} \preccurlyeq \mathcal{M}$ such that $\operatorname{acl}(a Z) \subset N, b \notin N$, and $N=\bigcup_{n \in \omega} A_{n}$ for a chain of some sets $A_{n}$.

In the initial step we consider the set $A_{0}=\operatorname{acl}(a Z)$ and renumber all consistent formulas of the form $\varphi(x, \bar{a}), \bar{a} \in A_{0}: \Phi_{0}=\left\{\varphi_{0, m}\left(x, \bar{a}_{m}\right) \mid m \in \omega\right\}$. According this numeration construct at most a countable set $A_{1}=\bigcup_{m \in \omega \cup\{-1\}} A_{1, m} \supset A_{0}$ with the condition $b \notin \operatorname{acl}\left(A_{1}\right)$. Let $A_{1,-1} \rightleftharpoons A_{0}$. If the set $A_{1, m-1}$ had been already defined and $\varphi_{0, m}\left(\mathcal{M}, \bar{a}_{m}\right) \cap A_{1, m-1} \neq \varnothing$ then we put $A_{1, m} \rightleftharpoons A_{1, m-1}$; if $\varphi_{0, m}\left(\mathcal{M}, \bar{a}_{m}\right) \cap A_{1, m-1}=\varnothing$ choose by Lemma 2.4 an element $c_{m} \in \varphi_{m}\left(\mathcal{M}, \bar{a}_{m}\right)$ such that $b \notin \operatorname{acl}\left(c_{m} A_{1, m-1}\right)$, and put $A_{1, m} \rightleftharpoons \operatorname{acl}\left(c_{m} A_{1, m-1}\right)$.

If at most a countable set $A_{n}$ had been already constructed, renumber all consistent formulas of the form $\varphi(x, \bar{a}), \bar{a} \in A_{n}: \Phi_{n}=\left\{\varphi_{n, m}\left(x, \bar{a}_{m}\right) \mid m \in \omega\right\}$. According to this enumeration construct at most a countable set $A_{n+1}=\bigcup_{m \in \omega \cup\{-1\}} A_{n+1, m} \supset A_{n}$ with the condition $b \notin \operatorname{acl}\left(A_{n+1}\right)$. Let $A_{n+1,-1} \rightleftharpoons A_{n}$. If the set $A_{n+1, m-1}$ had been already defined and $\varphi_{n, m}\left(\mathcal{M}, \bar{a}_{m}\right) \cap A_{n+1, m-1} \neq \varnothing$ then put $A_{n+1, m} \rightleftharpoons A_{n+1, m-1}$; if $\varphi_{n, m}\left(\mathcal{M}, \bar{a}_{m}\right) \cap A_{n+1, m-1}=$ $\varnothing$, choose by Lemma 2.4 an element $c_{m} \in \varphi_{n, m}\left(\mathcal{M}, \bar{a}_{m}\right)$ such that $b \notin \operatorname{acl}\left(c_{m} A_{n+1, m-1}\right)$ and put $A_{n+1, m} \rightleftharpoons \operatorname{acl}\left(c_{m} A_{n+1, m-1}\right)$.

By constructing the set $\bigcup_{n \in \omega} A_{n}$ forms a required universe $N$ of a countable model $\mathcal{N} \preccurlyeq \mathcal{M}$ such that $\operatorname{acl}(Z \cup\{a\}) \subseteq N$ and $b \notin N$.

Applying Lemma 2.4, we obtain the following lemma.

Lemma 2.5 Let $\mathcal{M}$ be an $\omega$-saturated model of a complete theory $T, \bar{a}, \bar{b} \in M, Z$ be the algebraic closure of some finite set in $\mathcal{M}$. If $(\operatorname{acl}(\bar{a} Z) \cap \operatorname{acl}(\bar{b} Z)) \backslash Z=\varnothing$ and $\varphi\left(x, \bar{a}^{\prime}\right)$ is a consistent formula, $\bar{a}^{\prime} \in \bar{a} Z$, then there is $c \in \varphi\left(\mathcal{M}, \bar{a}^{\prime}\right)$ such that $(\operatorname{acl}(\bar{a} c Z) \cap \operatorname{acl}(\bar{b} Z)) \backslash Z=\varnothing$. 
Theorem 2.6 Let $\mathcal{M}$ be an $\omega$-saturated model of a countable complete theory $T, Z$ be the algebraic closure of some finite set in $\mathcal{M}, a$ and $b$ be elements of $\mathcal{M}, a, b \notin Z$. The following are equivalent:

(1) the elements $a$ and $b$ are $Z$-separable in $H(\mathcal{M})$ by some sets $y$ and $z$ from $H(\mathcal{M})$ containing $Z$;

(2) the elements $a$ and $b$ are $Z$-separable in $H_{\omega_{1}}(\mathcal{M})$ by some sets $y$ and $z$ from $H_{\omega_{1}}(\mathcal{M})$ containing $Z$;

(3) $(\operatorname{acl}(a Z) \cap \operatorname{acl}(b Z)) \backslash Z=\varnothing$.

Proof. As in the proof of Theorem 2.3 it is sufficiently to prove the implication $(3) \Rightarrow(2)$. Assuming $(\operatorname{acl}(a Z) \cap \operatorname{acl}(b Z)) \backslash Z=\varnothing$, we construct by induction countable models $\mathcal{N}_{a}, \mathcal{N}_{b} \preccurlyeq$ $\mathcal{M}$ such that $\operatorname{acl}(a Z) \subseteq N_{a}, \operatorname{acl}(b Z) \subseteq N_{b},\left(N_{a} \cap N_{b}\right) \backslash Z=\varnothing, N_{a}=\bigcup_{n \in \omega} A_{n}$ for a chain of some sets $A_{n}$ and $N_{b}=\bigcup_{n \in \omega} B_{n}$ for a chain of some sets $B_{n}$.

In the initial step we consider the sets $A_{0}=\operatorname{acl}(a Z), B_{0}=\operatorname{acl}(b Z)$ and enumerate all consistent formulas of the form $\varphi(x, \bar{a}), \bar{a} \in A_{0}: \Phi_{0}=\left\{\varphi_{0, m}\left(x, \bar{a}_{m}\right) \mid m \in \omega\right\}$. According to this enumeration we construct at most countable set $A_{1}=\bigcup_{m \in \omega \cup\{-1\}} A_{1, m} \supset A_{0}$ with the condition $\left(\operatorname{acl}\left(A_{1}\right) \cap B_{0}\right) \backslash Z=\varnothing$. Let $A_{1,-1} \rightleftharpoons A_{0}$. If the set $A_{1, m-1}$ had been already defined and $\varphi_{0, m}\left(\mathcal{M}, \bar{a}_{m}\right) \cap A_{1, m-1} \neq \varnothing$, then put $A_{1, m} \rightleftharpoons A_{1, m-1}$; if $\varphi_{0, m}\left(\mathcal{M}, \bar{a}_{m}\right) \cap A_{1, m-1}=\varnothing$ then by Lemma 2.5 we choose an element $c_{m} \in \varphi_{m}\left(\mathcal{M}, \bar{a}_{m}\right)$ such that $\left(\operatorname{acl}\left(c_{m} A_{1, m-1}\right) \cap \operatorname{acl}\left(B_{0}\right)\right) \backslash$ $Z=\varnothing$ and put $A_{1, m} \rightleftharpoons \operatorname{acl}\left(c_{m} A_{1, m-1}\right)$.

If the set $A_{1}$ had been already defined, extend symmetrically the set $B_{0}$ to an algebraically closed set $B_{1}$ such that $B_{1} \supseteq Z$, all consistent formulas $\varphi(x, \bar{b}), \bar{b} \in B_{0}$, are realized in $B_{1}$ $\left(\operatorname{acl}\left(A_{1}\right) \cap \operatorname{acl}\left(B_{1}\right)\right) \backslash Z=\varnothing$.

If at most countable sets $A_{n}$ and $B_{n}$ had been already constructed, renumber all consistent formulas of the form $\varphi(x, \bar{a}), \bar{a} \in A_{n}: \Phi_{n}=\left\{\varphi_{n, m}\left(x, \bar{a}_{m}\right) \mid m \in \omega\right\}$. According to this numeration construct at most a countable set $A_{n+1}=\bigcup_{m \in \omega \cup\{-1\}} A_{n+1, m} \supset A_{n}$ with the condition $\left(\operatorname{acl}\left(A_{n+1}\right) \cap \operatorname{acl}\left(B_{1}\right)\right) \backslash Z=\varnothing$. Let $A_{n+1,-1} \rightleftharpoons A_{n}$. If the set $A_{n+1, m-1}$ had been already defined and $\varphi_{0, m}\left(\mathcal{M}, \bar{a}_{m}\right) \cap A_{n+1, m-1} \neq \varnothing$, then put $A_{n+1, m} \rightleftharpoons A_{n+1, m-1}$; if $\varphi_{0, m}\left(\mathcal{M}, \bar{a}_{m}\right) \cap A_{n+1, m-1}=\varnothing$, then by Lemma 2.5 choose an element $c_{m} \in \varphi_{n, m}\left(\mathcal{M}, \bar{a}_{m}\right)$ such that $\left(\operatorname{acl}\left(c_{m} A_{n+1, m-1}\right) \cap \operatorname{acl}\left(B_{n}\right)\right) \backslash Z=\varnothing$, and put $A_{n+1, m} \rightleftharpoons A_{n+1, m-1} \cup\left\{c_{m}\right\}$.

If we have the set $A_{n+1}$ then extend symmetrically the set $B_{n}$ to at most a countable set $B_{n+1}$ such that all consistent formulas $\varphi(x, \bar{b}), \bar{b} \in B_{n}$, are realized in $B_{n+1} \quad\left(\operatorname{acl}\left(A_{n+1}\right) \cap\right.$ $\left.\operatorname{acl}\left(B_{n+1}\right)\right) \backslash Z=\varnothing$.

By constructing the sets $\bigcup_{n \in \omega} A_{n}$ and $\bigcup_{n \in \omega} B_{n}$ form required universes $N_{a}$ and $N_{b}$ respectively of $Z$-separable countable models $\mathcal{N}_{a}, \mathcal{N}_{b} \preccurlyeq \mathcal{M}$ such that $a \in N_{a}$ and $b \in N_{b}$.

Combining proofs of Claims 1.4 1.10 and Theorems 2.3, 2.6. we obtain the following assertions.

Corollary 2.7 Let $\mathcal{M}$ be an $\omega$-saturated model of a small theory $T, Z$ be the algebraic closure of some finite set in $\mathcal{M}, a$ and $b$ be elements of $\mathcal{M}, a, b \notin Z$. The following are equivalent:

(1) the element a is $Z$-separable from the element $b$ in $H(\mathcal{M})$ by some set $y$ from $H(\mathcal{M})$ containing $Z$;

(2) the element $a$ is $Z$-separable from the element $b$ in $H_{\omega_{1}}(\mathcal{M})$ by some set $y$ from $H_{\omega_{1}}(\mathcal{M})$ containing $Z$; 
(3) the element a is $Z$-separable from the element $b$ in $H_{p}(\mathcal{M})$ by some set y from $H_{p}(\mathcal{M})$ containing $Z$;

(4) $b \notin \operatorname{acl}(a Z)$.

Corollary 2.8 Let $\mathcal{M}$ be an $\omega$-saturated model of a small theory $T, Z$ be the algebraic closure of some finite set in $\mathcal{M}, a$ and $b$ be elements of $\mathcal{M}, a, b \notin Z$. The following are equivalent:

(1) the elements $a$ and $b$ are $Z$-separable in $H(\mathcal{M})$ by some sets $y$ and $z$ from $H(\mathcal{M})$ containing $Z$;

(2) the elements $a$ and $b$ are $Z$-separable in $H_{\omega_{1}}(\mathcal{M})$ by some sets $y$ and $z$ from $H_{\omega_{1}}(\mathcal{M})$ containing $Z$;

(3) the elements $a$ and $b$ are separable in $H_{p}(\mathcal{M})$ by some sets $y$ and $z$ from $H_{p}(\mathcal{M})$ containing $Z$;

(4) $(\operatorname{acl}(a Z) \cap \operatorname{acl}(b Z)) \backslash Z=\varnothing$.

Theorem 2.9 Let $\mathcal{M}$ be a $\lambda$-saturated model of a complete theory $T, \lambda \geq \max \{|\Sigma(T)|, \omega\}$, $A$ and $B$ be nonempty sets in $\mathcal{M}$ having cardinalities $<\lambda, Z$ be the algebraic closure of some set of cardinality $<\lambda$ in $\mathcal{M}$. The following are equivalent:

(1) the set $A$ is $Z$-separable from the set $B$ in $H(\mathcal{M})$ by some set y from $H(\mathcal{M})$ containing $Z$;

(2) the set $A$ is $Z$-separable from the set $B$ in $H_{\lambda}(\mathcal{M})$ by some set $y$ from $H_{\lambda}(\mathcal{M})$ containing $Z$;

(3) $(\operatorname{acl}(A \cup Z) \cap B) \backslash Z=\varnothing$.

Theorem 2.10 Let $\mathcal{M}$ be a $\lambda$-saturated model of a complete theory $T, \lambda \geq \max \{|\Sigma(T)|, \omega\}$, $A$ and $B$ be nonempty sets in $\mathcal{M}$ having cardinalities $<\lambda, Z$ be the algebraic closure of some set of cardinality $<\lambda$ in $\mathcal{M}$. The following are equivalent:

(1) the sets $A$ and $B$ are $Z$-separable in $H(\mathcal{M})$ by some sets $y$ and $z$ from $H(\mathcal{M})$ containing $Z$;

(2) the sets $A$ and $B$ are $Z$-separable in $H_{\lambda}(\mathcal{M})$ by some sets $y$ and $z$ from $H_{\lambda}(\mathcal{M})$ containing $Z$;

(3) $(\operatorname{acl}(A \cup Z) \cap \operatorname{acl}(B \cup Z)) \backslash Z=\varnothing$.

Corollary 2.11 Let $\mathcal{M}$ be an $\omega$-saturated model of a small theory $T, A$ and $B$ be finite nonempty sets in $\mathcal{M}, Z$ be the algebraic closure of some finite set in $\mathcal{M}$. The following are equivalent:

(1) the set $A$ is $Z$-separable from the set $B$ in $H(\mathcal{M})$ by some set $y$ from $H(\mathcal{M})$ containing $Z$;

(2) the set $A$ is $Z$-separable from the set $B$ in $H_{\omega_{1}}(\mathcal{M})$ by some set $y$ from $H_{\omega_{1}}(\mathcal{M})$ containing $Z$;

(3) the set $A$ is $Z$-separable from the set $B$ in $H_{p}(\mathcal{M})$ by some set $y$ from $H_{p}(\mathcal{M})$ containing $Z$;

(4) $(\operatorname{acl}(A \cup Z) \cap B) \backslash Z=\varnothing$.

Corollary 2.12 Let $\mathcal{M}$ be an $\omega$-saturated model of a small theory $T, A$ and $B$ be finite nonempty sets in $\mathcal{M}, Z$ be the algebraic closure of some finite set in $\mathcal{M}$. The following are equivalent:

(1) the sets $A$ and $B$ are $Z$-separable in $H(\mathcal{M})$ by some sets $y$ and $z$ from $H(\mathcal{M})$ containing $Z$; 
(2) the sets $A$ and $B$ are $Z$-separable in $H_{\omega_{1}}(\mathcal{M})$ by some sets $y$ and $z$ from $H_{\omega_{1}}(\mathcal{M})$ containing $Z$;

(3) the sets $A$ and $B$ are $Z$-separable in $H_{p}(\mathcal{M})$ by some sets $y$ and $z$ from $H_{p}(\mathcal{M})$ containing $Z$;

(4) $(\operatorname{acl}(A \cup Z) \cap \operatorname{acl}(B \cup Z)) \backslash Z=\varnothing$.

\section{On separability in hypergraphs of models of ordered theo- ries}

Definition 3.1 [1, 15] Let $p_{1}\left(x_{1}\right), \ldots, p_{n}\left(x_{n}\right) \in S_{1}(T)$. A type $q\left(x_{1}, \ldots, x_{n}\right) \in S(T)$ is called $\left(p_{1}, \ldots, p_{n}\right)$-type if $q\left(x_{1}, \ldots, x_{n}\right) \supseteq \bigcup_{i=1}^{n} p_{i}\left(x_{i}\right)$. The set of all $\left(p_{1}, \ldots, p_{n}\right)$-types of a theory $T$ is denoted by $S_{p_{1}, \ldots, p_{n}}(T)$. A countable theory $T$ is called almost $\omega$-categorical if for any types $p_{1}\left(x_{1}\right), \ldots, p_{n}\left(x_{n}\right) \in S(T)$ there exist only finitely many types $q\left(x_{1}, \ldots, x_{n}\right) \in S_{p_{1}, \ldots, p_{n}}(T)$.

Theorem 3.2 Let $T$ be an almost $\omega$-categorical quite o-minimal theory, $\mathcal{M}$ be an $\omega$-saturated model of the theory $T, Z$ be the algebraic closure of some finite set in $\mathcal{M}, a, b \in M \backslash Z$. Then the following conditions are equivalent:

(1) $a$ is $Z$-separable from $b$ in $\mathcal{H}(\mathcal{M})$ by some set $y$ from $H(\mathcal{M})$ containing $Z$;

(2) $b$ is $Z$-separable from a in $\mathcal{H}(\mathcal{M})$ by some set $y$ from $H(\mathcal{M})$ containing $Z$;

(3) the elements $a$ and $b$ are $Z$-separable in $H(\mathcal{M})$ by some sets $y$ and $z$ from $H(\mathcal{M})$ containing $Z$;

(4) $a \notin \operatorname{dcl}(\{b Z\})$;

(5) $b \notin \operatorname{dcl}(\{a Z\})$.

(6) $(\operatorname{dcl}(a Z) \cap \operatorname{dcl}(b Z)) \backslash Z=\varnothing$.

Proof of Theorem 3.2, By Proposition 3.9 [10] Exchange Principle for algebraic closure holds. By linear ordering of the model $\mathcal{M} \operatorname{dcl}(A)=\operatorname{acl}(A)$ for any $A \subseteq M$. Then by proofs of Theorems 2.3 and 2.6 we have an equivalence of the conditions (1)-(6).

Remark 3.3 1. Theorem 3.2 remains true for an arbitrary theory satisfying both Exchange Principle for algebraic closure and the condition $\operatorname{dcl}(A)=\operatorname{acl}(A)$ for any $A \subseteq M$.

2. If Exchange Principle for algebraic closure holds and the condition $\operatorname{dcl}(A)=\operatorname{acl}(A)$ for any $A \subseteq M$ doesn't hold, Theorem 3.2 remains true if we replace dcl by acl.

3. If the condition $\operatorname{dcl}(A)=\operatorname{acl}(A)$ for any $A \subseteq M$ holds and Exchange Principle for algebraic closure doesn't hold, Theorem 3.2 splits into three independent statements $(1) \Leftrightarrow(5),(2) \Leftrightarrow(4),(3) \Leftrightarrow(6)$.

Theorem 3.2 immediately implies the following

Corollary 3.4 Let $T$ be an almost $\omega$-categorical quite o-minimal theory, $\mathcal{M}$ be an $\omega$-saturated model of the theory $T, a, b \in M \backslash d c l(\varnothing)$. Then the following conditions are equivalent:

(1) $a$ is separable from $b$ in $\mathcal{H}(\mathcal{M})$;

(2) $b$ is separable from a in $\mathcal{H}(\mathcal{M})$;

(3) $a \notin \operatorname{dcl}(\{b\})$;

(4) $b \notin \operatorname{dcl}(\{a\})$. 
Example 3.5 [1] Let $\mathcal{M}=\left\langle M ;<, P_{1}^{1}, P_{2}^{1}, f^{1}\right\rangle$ be a linearly ordered structure such that $M$ is the disjoint union of interpretations of unary predicates $P_{1}$ and $P_{2}$ so that $P_{1}(\mathcal{M})<P_{2}(\mathcal{M})$. We identify an interpretation of $P_{2}$ with the set of rational numbers $\mathbb{Q}$, ordered as usual, and $P_{1}$ with $\mathbb{Q} \times \mathbb{Q}$, ordered lexicographically. The symbol $f$ is interpreted by a partial unary function with $\operatorname{Dom}(f)=P_{1}(\mathcal{M})$ and Range $(f)=P_{2}(\mathcal{M})$ and is defined by the equality $f((n, m))=n$ for all $(n, m) \in \mathbb{Q} \times \mathbb{Q}$.

It is known that $\mathcal{M}$ is a countably categorical weakly o-minimal structure, and $T h(\mathcal{M})$ is not quite o-minimal. Take arbitrary $a \in P_{1}(\mathcal{M}), b \in P_{2}(\mathcal{M})$ such that $f(a)=b$. Then we obtain that $a$ is separable from $b$ in $\mathcal{H}(\mathcal{M})$, but $b$ is not separable from $a$ in $\mathcal{H}(\mathcal{M})$.

Proposition 3.6 Let $T$ be an almost $\omega$-categorical quite o-minimal theory, $\mathcal{M} \models T, A=$ $\left\{a_{1}, \ldots, a_{n_{1}}\right\}, B=\left\{b_{1}, \ldots, b_{n_{2}}\right\} \subseteq M$ for some positive $n_{1}, n_{2}<\omega$. Then the following conditions are equivalent:

(1) $A$ and $B$ are separable from each other in $\mathcal{H}(\mathcal{M})$;

(2) $\operatorname{dcl}(A) \cap \operatorname{dcl}(B)=\emptyset$.

(3) $\operatorname{dcl}\left(\left\{a_{i}\right\}\right) \cap \operatorname{dcl}\left(\left\{b_{j}\right\}\right)=\emptyset$ for any $1 \leq i \leq n_{1}, 1 \leq j \leq n_{2}$.

Proof of Proposition 3.6. (1) $\Rightarrow(2)$ Let $A$ be separable from $B$ in $\mathcal{H}(\mathcal{M})$. This means that there is $\mathcal{M}_{1} \prec \mathcal{M}$ such that $A \subseteq M_{1}$ and $B \cap M_{1}=\emptyset$. Then we have: $\operatorname{dcl}(A) \subseteq M_{1}$, whence we obtain that $\operatorname{dcl}(A) \cap B=\emptyset$. Similarly, by the condition of separability of $B$ from $A$ in $\mathcal{H}(\mathcal{M})$ it can be established that $\operatorname{dcl}(B) \cap A=\emptyset$.

Assume the contrary: $\operatorname{dcl}(A) \cap \operatorname{dcl}(B) \neq \emptyset$. Consequently, there is $c \in M$ such that $c \in \operatorname{dcl}(A)$ and $c \in \operatorname{dcl}(B)$. But then by binarity of $T h(\mathcal{M})$ there exist $a \in A$ and $b \in B$ such that $c \in \operatorname{dcl}(\{a\})$ and $c \in \operatorname{dcl}(\{b\})$. By holding Exchange Principle for algebraic closure we obtain that $b \in \operatorname{dcl}(\{a\})$. The last contradicts the condition $\operatorname{dcl}(A) \cap B=\emptyset$.

$(2) \Rightarrow(1)$ In this case we assert that $M_{1}:=M \backslash \operatorname{dcl}(A)$ and $M_{2}:=M \backslash \operatorname{dcl}(B)$ are universes of elementary submodels of the model $\mathcal{M}$.

$(2) \Leftrightarrow(3)$ By binarity of $T h(\mathcal{M})$.

Proposition 3.7 Let $T$ be an almost $\omega$-categorical quite o-minimal theory, $\mathcal{M} \models T, Z=$ $\operatorname{dcl}(\emptyset), A=\left\{a_{1}, \ldots, a_{n_{1}}\right\}, B=\left\{b_{1}, \ldots, b_{n_{2}}\right\} \subseteq M$ for some positive $n_{1}, n_{2}<\omega$ so that $A \cap Z=B \cap Z=\emptyset$. Then the following conditions are equivalent:

(1) $A$ and $B$ are $Z$-separable in $\mathcal{H}(\mathcal{M})$;

(2) $\operatorname{dcl}(A) \cap \operatorname{dcl}(B)=Z$.

(3) $\operatorname{dcl}\left(\left\{a_{i}\right\}\right) \cap \operatorname{dcl}\left(\left\{b_{j}\right\}\right)=Z$ for any $1 \leq i \leq n_{1}, 1 \leq j \leq n_{2}$.

Proof of Proposition 3.7 (1) $\Rightarrow(2)$ Let $A$ and $B$ be $Z$-separable in $\mathcal{H}(\mathcal{M})$. Then there exist $\mathcal{M}_{1}, \mathcal{M}_{2} \prec \mathcal{M}$ such that $\left(\mathcal{M}_{1} \cap \mathcal{M}_{2}\right) \backslash Z=\emptyset, A \subseteq M_{1}$ and $B \subseteq M_{2}$. Consequently, $\operatorname{dcl}(A) \cap \operatorname{dcl}(B) \subseteq \mathcal{M}_{1} \cap \mathcal{M}_{2}$. Then $[\operatorname{dcl}(A) \cap \operatorname{dcl}(B)] \backslash Z=\emptyset$, whence $\operatorname{dcl}(A) \cap \operatorname{dcl}(B)=Z$.

$(2) \Rightarrow(1)$ In this case we assert that $M_{1}:=[M \backslash \operatorname{dcl}(A)] \cup Z$ and $M_{2}:=[M \backslash \operatorname{dcl}(B)] \cup Z$ are universes of elementary submodels of the model $\mathcal{M}$.

Arguments for Propositions 1.11 and 3.6 imply the following

Proposition 3.8 Let $T$ be an almost $\omega$-categorical quite o-minimal theory, $\mathcal{M} \models T, \emptyset \neq$ $A, B \subseteq M, \mathcal{M}$ be $|A \cup B|^{+}$-saturated. Then $A$ and $B$ are separable from each other in $\mathcal{H}(\mathcal{M})$ if and only if the following conditions hold:

(1) $\operatorname{dcl}(\{a\}) \cap \operatorname{dcl}(\{b\})=\emptyset$ for any $a \in A$ and $b \in B$;

(2) For any isolated type $p \in S_{1}(\emptyset), p(\mathcal{M}) \backslash \operatorname{dcl}(A) \neq \emptyset$ and $p(\mathcal{M}) \backslash \operatorname{dcl}(B) \neq \emptyset$. 
Corollary 3.9 Let $T$ be an almost $\omega$-categorical quite o-minimal theory, $\mathcal{M} \models T, Z=\operatorname{dcl}(\emptyset)$, $A, B$ be non-empty subsets of $M$ such that $A \cap Z=B \cap Z=\emptyset, \mathcal{M}$ be $|A \cup B|^{+}$-saturated. Then $A$ and $B$ are $Z$-separable in $\mathcal{H}(\mathcal{M})$ if and only if the following conditions hold:

(1) $\operatorname{dcl}(\{a\}) \cap \operatorname{dcl}(\{b\})=Z$ for any $a \in A$ and $b \in B$;

(2) For any isolated type $p \in S_{1}(\emptyset), p(\mathcal{M}) \backslash \operatorname{dcl}(A) \neq \emptyset$ and $p(\mathcal{M}) \backslash \operatorname{dcl}(B) \neq \emptyset$.

Arguments for Propositions 1.11 and 3.6 as well as Theorem 2.10 imply the following

Proposition 3.10 Let $T$ be an almost $\omega$-categorical quite o-minimal theory, $\mathcal{M} \models T$ be $\lambda$ saturated, $\lambda \geq \max \{|\Sigma(T)|, \omega\}, A$ and $B$ be nonempty sets in $\mathcal{M}$ having cardinalities $<\lambda$, $Z$ be the algebraic closure of some set of cardinality $<\lambda$ in $\mathcal{M}$. Then the following are equivalent:

(1) $A$ and $B$ are $Z$-separable in $\mathcal{H}(\mathcal{M})$;

(2) $(\operatorname{dcl}(a Z) \cap \operatorname{dcl}(b Z)) \backslash Z=\emptyset$ for any $a \in A$ and $b \in B$.

Acknowledgements. This research was partially supported by Committee of Science in Education and Science Ministry of the Republic of Kazakhstan (Grant No. AP05132546) and Russian Foundation for Basic Researches (Project No. 17-01-00531-a).

\section{References}

[1] S.V. Sudoplatov, Classification of countable models of complete theories. - Novosibirsk : Novosibirsk State Technical University Publishing House, 2014.

[2] S.V. Sudoplatov, On acyclic hypergraphs of minimal prime models // Siberian Mathematical Journal. - 2001. - Vol. 42, issue 6, pp. 1408-1412.

[3] S.V. Sudoplatov, Hypergraphs of prime nmodels and distributions of countable models of small theories // J. Math. Sciences, 2010, vol. 169, issue 5, pp. 680-695.

[4] K.A. Baikalova, On some hypergraphs of prime models and limit models generated by them // Algebra and Model Theory, 7. Collection of works edited by A.G. Pinus, K.N. Ponomaryov, S.V. Sudoplatov. - Novosibirsk : Novosibirsk State Technical University Publishing House, 2009, pp. 6-17.

[5] S.V. Sudoplatov, On the separability of elements and sets in hypergraphs of models of a theory // Bulletin of Karagandy University. Series Mathematics, 2016, vol. 82, issue 2, pp. 113-120.

[6] B.Sh. Kulpeshov, S.V. Sudoplatov, On hypergraphs of prime models for quite o-minimal theories with small number of countable models // Annual Scientific April Conference of Institute of Mathematics and Mathematical Modelling devoted to Science Day and Scientific Seminar "Differential operators and modelling complex systems" (DOMCS2017) devoted to 70-th anniversary of professor M.T. Dzhenaliev, Almaty, 7-8 April 2017: abstracts of talks. - Almaty: IMMM, 2017. — pp. 30-32.

[7] S.V. Sudoplatov, Derivative Structures in Model Theory and Group Theory // International Conference "Actual Problems of Pure and Applied Mathematics" devoted to 100-th anniversary of academician A.D. Taimanov, Almaty, 22-25 August 2017: abstracts of talks. - Almaty: IMMM, 2017, pp. 76-79. 
[8] B.Sh. Kulpeshov, S.V. Sudoplatov, On decomposition of hypergraphs of models of a theory. Appendix to theories of unars // Sintax and Semantics of Logical Systems: Materials of 5-th Russian School-Seminar. - Ulan-Ude: Buryatsky State University Publishing House, 2017, pp. 52-56.

[9] R. Engelking, General Topology, Revised and completed edition, Heldermann Verlag (Sigma series in pure mathematics, Vol. 6), Berlin, 1989.

[10] B.Sh. Kulpeshov, S.V. Sudoplatov, Linearly ordered theories which are nearly countably categorical // Mathematical Notes, 2017, volume 101, No. 3, pp. 475-483.

[11] H.D. Macpherson, D. Marker, and C. Steinhorn, Weakly o-minimal structures and real closed fields // Transactions of The American Mathematical Society, 352 (2000), pp. $5435-5483$.

[12] B.S. Baizhanov, Expansion of a model of a weakly o-minimal theory by a family of unary predicates // The Journal of Symbolic Logic, 66 (2001), pp. 1382-1414.

[13] B.Sh. Kulpeshov, Convexity rank and orthogonality in weakly o-minimal theories // News of National Academy of Sciences of the Republic of Kazakhstan, series physicsmathematics, 227 (2003), pp. 26-31.

[14] B.Sh. Kulpeshov, S.V. Sudoplatov, Vaught's conjecture for quite o-minimal theories // Annals of Pure and Applied Logic, 2017, vol. 168, issue 1, pp. 129-149.

[15] K. Ikeda, A. Pillay, A. Tsuboi, On theories having three countable models // Mathematical Logic Quarterly, vol. 44, issue 2, 1998, pp. 161-166. 